\title{
Forecasting US interest rates and business cycle with a nonlinear regime switching VAR model
}

\section{Nyberg, Henri}

2018-01

Nyberg , H 2018, ' Forecasting US interest rates and business cycle with a nonlinear regime switching VAR model ' , Journal of Forecasting, vol. 37 , no. 1, pp. 1-15 . https://doi.org/10.1002/for.2458

http://hdl.handle.net/10138/312741

https://doi.org/10.1002/for.2458

acceptedVersion

Downloaded from Helda, University of Helsinki institutional repository.

This is an electronic reprint of the original article.

This reprint may differ from the original in pagination and typographic detail.

Please cite the original version. 
This is the peer reviewed version of the following article: Nyberg, H. (2017), Forecasting U.S. Interest Rates and Business Cycle with a Nonlinear Regime Switching VAR Model, Journal of Forecasting (forthcoming), which has been published in final form at DOI: http://dx.doi.org/10.1002/for.2458. This article may be used for non-commercial purposes in accordance with Wiley Terms and Conditions for Self-Archiving.

\title{
Forecasting U.S. Interest Rates and Business Cycle
}

\section{with a Nonlinear Regime Switching VAR Model}

\author{
Henri Nyberg* \\ University of Turku, Department of Mathematics and Statistics \\ University of Helsinki, Department of Political and Economic Studies, \\ Economics
}

\begin{abstract}
This paper introduces a regime switching vector autoregressive model with time-varying regime probabilities, where the regime switching dynamics is described by an observable binary response variable predicted simultaneously with the variables subject to regime changes. Dependence on the observed binary variable distinguishes the model from various previously proposed multivariate regime switching models, facilitating a handy simulation-based multistep forecasting method. An empirical application shows a strong bidirectional predictive linkage between the U.S. interest rates and the NBER business cycle recession and expansion periods. Due to the predictability of the business cycle regimes, the proposed model yields superior out-of-sample forecasts of the U.S. short-term interest rate and the term spread compared with the linear and nonlinear VAR models, including the Markov switching VAR model.
\end{abstract}

JEL classification numbers: C34, C35, C53, E43, E44.

Keywords: Nonlinear vector autoregression, Probit model, Turning points, Financial markets, Simulation.

*Address: Department of Mathematics and Statistics, 20014 University of Turku, Finland. E-mail: henri.nyberg@utu.fi, Tel: +358 503182262. 


\section{Introduction}

Nonlinear econometric modeling has heavily been based on regime switching mechanisms allowing for parameter coefficients to switch between different states of the world (e.g., business cycle recessions and expansions, bear and bull stock markets, monetary policy regimes and also some rare events such as financial crises). The previous literature on multivariate models has adopted several different regime switching specifications including Sola and Driffill (1994), Krolzig (1997), Ang and Bekaert (2002a,b), Guidolin and Timmermann (2006), Dueker et al. (2011) and Henkel et al. (2011), among others. In this literature, the regime switching mechanism is typically specified as a latent (unobserved) process with underlying regime probabilities which may be functions of the lagged endogenous or exogenous variables determining the economic forces driving the regime switches. However, in line with nonlinear models in general, the out-of-sample forecasting performances of these models have often been found disappointing (see, e.g., the discussion in Dacco and Satchell (1999) and Clements et al. (2004)).

In this study, we consider a regime switching vector autoregressive (VAR) model, where the regime is determined by an observed qualitative response (QR) variable predicted simultaneously with the variables subject to regime switches and, hence, permitting the method implementable in real time forecasting. The joint model is, for simplicity, referred to the QR-VAR model. The use of the qualitative response model yields time-varying regime probabilities between the observed regimes making the QR-VAR model much easier to work with and, in particular, construct forecasts than the multivariate regime switching models with latent regimes. Following the large majority of the previous studies, we restrict ourselves to the two regime case, that is the qualitative variable is binary throughout this paper. In our empirical application, the binary variable is the state of the U.S. business cycle measured in terms of the official NBER business cycle turning points. A multinomial case (i.e. multiple regimes) is a straightforward extension to our model, provided that the observed qualitative time series determining the 
regimes is available.

The dependence on the observed qualitative dependent variable distinguishes the QR-VAR model from the commonly used Markov switching VAR and related models, which, of course, have their own advantages. They are more general in a sense that the latent regimes are extracted based on statistical grounds while in our approach the dynamics are driven by the observed qualitative variable. However, as long as the objective is to link the latent regime dynamics directly to some well-established regimes such as the NBER business cycle periods, which has typically been the case in various applications of Markov switching models, the QR-VAR model offers a much simpler way to estimate the parameters and construct multiperiod forecasts. This is due to the fact that the resulting conditional probabilities of the regimes can be constructed with a binary response model simplifying parameter estimation carried out with the method of maximum likelihood. This approach circumvents the difficulties reported in the parameter estimation of various previous models (see, e.g., Gray, 1996; Simpson et al., 2001; Ang and Bekaert, 2002a,b) where estimation requires the filtration of the latent regimes (see also the discussion in Filardo and Gordon, 1998).

In general, if the values of a qualitative dependent variable, such as the state of the business cycle, are predictable, then so are the regime switches in the QRVAR model. This should lead to superior forecast performance compared with the single-regime VAR model (provided there are regime switches in the VAR process). The QR-VAR model is designed to produce dynamic iterative forecasts constructed sequentially for the binary (qualitative) and continuous variables. We propose a simulation-based method to obtain multiperiod forecasts as closed-form forecasting formulae are generally not available. The examined Monte Carlo forecasting experiments show that the proposed method is not, however, computationally burdensome and it leads forecasting gains over the single-regime VAR model. An important advantage of our model is that it facilitates multistep forecasting while in the previous univariate and multivariate regime switching models, with dependence on latent regimes and time-varying transition probabilities, only one-period-ahead 
forecasts have been considered so far (see, e.g., Filardo, 1994; Perez-Quiros and Timmermann, 2000; Simpson et al., 2001; Ang and Bekaert, 2002a).

In addition to the regime switching perspective emphasized above, the QR-VAR model adds to very scant literature on models where continuous real-valued and qualitative dependent time series are modeled jointly (see the related models with a similar structure in Hamilton and Jordà (2002) and Nyberg (2012)). Dueker (2005) and Fornari and Lemke (2010) are two rare exceptions where the VAR model is augmented with a latent variable determining the values of the considered binary time series. Our model differs from their models in various ways: In particular, Dueker (2005) and Fornari and Lemke (2010) do not allow a regime switches in their VAR models, and the latter also employs a commonly used static probit model for the binary variable. In line with the univariate models of Rydberg and Shephard (2003), Benjamin et al. (2003) and Kauppi and Saikkonen (2008), we use a dynamic binary response model as a part of the model leading to the model specification where estimation and forecasting is easier than in the dynamic model of Dueker (2005).

We apply the QR-VAR model to forecast the U.S. interest rates and the state of the business cycle in real time. As an example, Ang and Piazzesi (2003), Bansal et al. (2004) and Huse (2011) have shown that macroeconomic factors measuring real economic activity can help to predict future movements in the yield curve. In contrast, Estrella and Mishkin (1998) and Rudebusch and Williams (2009), among others, have found that the term spread between the long-term and shortterm interest rates is the main leading indicator of the future state of the business cycle. Interestingly, almost all previous studies have concentrated on these one-way linkages while, e.g., Estrella (2005) and Diebold et al. (2006) are supportive for a bidirectional relationship, without allowing for regime switches in the interest rates. In this study, instead of using the ex post observations of the U.S. business cycle regimes, the regimes are predicted simultaneously with the interest rate variables. To the best of our knowledge, this type of regime switching forecasting approach has not been considered before in the literature. 
Our empirical results provide several interesting insights. In particular, strong evidence of business cycle-specific effects in the bivariate system of the U.S. shortterm interest rate and the term spread is obtained. The dynamics of the short rate are closely dependent on the NBER expansion and recession periods of the U.S. economy whereas the lags of interest rate variables predict the state of the business cycle. Furthermore, and most importantly, due to the obtained predictability of business cycle turning points in real time, the out-of-sample forecasts of the QRVAR model outperform those of the single-regime VAR model for the term spread and, especially, the short-term interest rate. That is also the case when comparing the forecasting performance to the existing regime switching models, including the Markov switching VAR model.

The rest of the paper is organized as follows. Section 2 introduces our regime switching VAR model. Parameter estimation and computation of forecasts, including the proposed simulation-based forecasting method, are considered in Section 3. The forecasting results containing analyses on the bidirectional predictive linkages and feedback mechanisms between the U.S. interest rates and business cycle are reported in Section 4. Finally, Section 5 concludes.

\section{Model}

Consider the observable time series $s_{t}$ and $\mathbf{y}_{t}, t=1,2, \ldots, T$, where $s_{t}$ is a qualitative response variable and $\mathbf{y}_{t}=\left[y_{1 t}, \ldots, y_{K t}\right]^{\prime}$ is a $K \times 1$ random vector of real-valued continuous variables. Thus, for simplicity, we refer our model as the Qualitative Response Vector AutoRegressive (QR-VAR) model. Throughout this paper, we concentrate on the case where $s_{t}$ is binary taking values 0 or 1 (i.e. two regimes), but a multinomial (multiple regime) dependent variable is a straightforward extension to this case.

For notational convenience, the variables are collected to the vector

$$
\boldsymbol{z}_{t}=\left[\begin{array}{ll}
s_{t} & \mathbf{y}_{t}^{\prime}
\end{array}\right]^{\prime}
$$


The novel idea is to construct a regime switching VAR model where the regimes are determined by the observable binary variable $s_{t}$. The regime switching VAR model can be written as

$$
\mathbf{y}_{t}=s_{t}\left(\boldsymbol{w}_{1}+\sum_{i=1}^{p_{1}} \boldsymbol{A}_{i, 1} \mathbf{y}_{t-i}+\mathbf{e}_{1 t}\right)+\left(1-s_{t}\right)\left(\boldsymbol{w}_{0}+\sum_{i=1}^{p_{0}} \boldsymbol{A}_{i, 0} \mathbf{y}_{t-i}+\mathbf{e}_{0 t}\right),
$$

where depending on whether $s_{t}$ takes the value 0 or $1, \mathbf{y}_{t}$ follows a different VAR model. In other words, if $s_{t}=1$, we are in the regime 1 and otherwise $\left(s_{t}=0\right)$ in the regime 0 . The constant terms $\boldsymbol{w}_{j}$, coefficient matrices $\boldsymbol{A}_{i, j}, i=1, \ldots, p_{j}$, and the error terms $\mathbf{e}_{j t}, j=0,1$, are all regime-specific allowing for flexible and different dynamics in two regimes. Model (2) encompasses the conventional $\operatorname{VAR}(p)$ model when $p_{0}=p_{1}, \mathbf{e}_{0 t}=\mathbf{e}_{1 t}$ and all the corresponding parameters are the same irrespective of the regime $s_{t}$.

In model (2), the error terms $\mathbf{e}_{0 t}$ and $\mathbf{e}_{1 t}$ are assumed to follow multivariate normal distributions with zero means and possibly different covariance matrices $\boldsymbol{\Sigma}_{0}$ and $\boldsymbol{\Sigma}_{1}$ depending on the regime. Thus, we write

$$
\mathbf{e}_{j t}=\boldsymbol{\Sigma}_{j}^{1 / 2} \mathbf{e}_{t}, j=0,1, \quad \mathbf{e}_{t} \sim \operatorname{NID}\left(\mathbf{0}, \mathbf{I}_{K}\right)
$$

and assume that $\mathbf{e}_{t}$ and $\Omega_{t-1}$ are independent with $\Omega_{t-1}=\left\{\boldsymbol{z}_{t-1}, \boldsymbol{z}_{t-2}, \ldots, \boldsymbol{z}_{1}\right\}$ denoting the information set containing the lags of $\mathbf{y}_{t}$ and $s_{t}$ (see (1)) at time $t-1$. Furthermore, $\mathbf{e}_{t}$ and $s_{t}$ are assumed to be independent conditional on $\Omega_{t-1}$.

Throughout this paper, we assume that in (2) the contemporaneous value of $s_{t}$ has an effect on $\mathbf{y}_{t}$, but not vice versa (cf. the model of Nyberg, 2012). Although the main interest is in the regime switching VAR model (2), a model for the binary variable $s_{t}$ is also needed to forecast the future values of $\mathbf{y}_{t}$ (see Section 3 ). Conditional on the information set $\Omega_{t-1}, s_{t}$ follows a Bernoulli distribution

$$
s_{t} \mid \Omega_{t-1} \sim B\left(p_{t}\right) .
$$

In this expression, $p_{t}$ is the conditional expectation of $s_{t}$ (denoted by $E_{t-1}\left(s_{t}\right)$ ) 
or equivalently the conditional probability of the outcome $s_{t}=1$ (denoted by $\left.P_{t-1}\left(s_{t}=1\right)\right)$

$$
p_{t}=E_{t-1}\left(s_{t}\right)=P_{t-1}\left(s_{t}=1\right)=\Phi\left(\pi_{t}\right),
$$

where $\Phi(\cdot)$ is a standard normal cumulative distribution function leading to the probit model and $\pi_{t}$ is a linear function of variables included in the information set $\Omega_{t-1}$. An alternative to the probit model, a logit model, is obtained by replacing $\Phi(\cdot)$ in (5) with the logistic function.

To complete the model for the binary variable $s_{t}$, we specify

$$
\pi_{t}=\nu+a \pi_{t-1}+\mathbf{x}_{t-1}^{\prime} \mathbf{b}
$$

where $|a|<1$ and $\nu$ is a constant term. This model was suggested by Kauppi and Saikkonen (2008) in the context of univariate binary time series models (see also Rydberg and Shephard, 2003; Benjamin et al., 2003). For simplicity, we restrict ourselves to the case where the predictors included in the vector $\mathbf{x}_{t-1}$ are the lagged values of $\mathbf{y}_{t}$. For example, if $K=2$, then we set $\mathbf{x}_{t-1}=\left[\begin{array}{lll}y_{1, t-k_{1}} & y_{2, t-k_{2}}\end{array}\right]^{\prime}$ with $k_{1}$ and $k_{2} \geq 1$. By recursive substitutions, and assuming $|a|<1, \pi_{t}$ will depend on the whole lagged history of the predictive variables:

$$
\pi_{t}=\sum_{i=1}^{\infty} a^{i-1}\left(\nu+\mathbf{x}_{t-i}^{\prime} \boldsymbol{b}\right) .
$$

The univariate probit model is obtained when the predictors in $\mathbf{x}_{t-1}$ are treated as exogenous predictive variables. In the previous business cycle recession forecasting literature, dynamic univariate models, such as model (6) (see, e.g., Kauppi and Saikkonen, 2008, Nyberg, 2010), have been found to outperform the usual static model obtained when $a=0$ in (6) (see, e.g., Estrella and Mishkin, 1998; Sensier et al., 2004; Wright, 2006).

The expressions (2), (3), (5) and (6) define together the QR-VAR $\left(p_{0}, p_{1}\right)$ model, where $p_{0}$ and $p_{1}$ denote the lag lengths of $\mathbf{y}_{t}$ in the regimes of model (2). Equation (2) shows the regime switching mechanism of the model but in forecast compu- 
tation in Section 3, we need the conditional expectation of $\mathbf{y}_{t}$ given $\Omega_{t-1}$. This results in

$$
\begin{aligned}
E_{t-1}\left(\mathbf{y}_{t}\right) & =E_{t-1}\left[s_{t}\left(\boldsymbol{w}_{1}+\sum_{i=1}^{p_{1}} \boldsymbol{A}_{i, 1} \mathbf{y}_{t-i}+\mathbf{e}_{1 t}\right)+\left(1-s_{t}\right)\left(\boldsymbol{w}_{0}+\sum_{i=1}^{p_{0}} \boldsymbol{A}_{i, 0} \mathbf{y}_{t-i}+\mathbf{e}_{0 t}\right)\right] \\
& =p_{t} \boldsymbol{\mu}_{1 t}+\left(1-p_{t}\right) \boldsymbol{\mu}_{0 t}
\end{aligned}
$$

where $\boldsymbol{\mu}_{j t}=\boldsymbol{w}_{j}+\sum_{i=1}^{p_{j}} \boldsymbol{A}_{i, j} \mathbf{y}_{t-i}, j=0,1$, and the law of iterated expectations and the assumptions made in (3) imply

$$
\begin{aligned}
E_{t-1}\left(s_{t} \mathbf{e}_{j t}\right) & =E_{t-1}\left[E\left(s_{t} \mathbf{e}_{j t} \mid s_{t}, \Omega_{t-1}\right)\right] \\
& =E_{t-1}\left[s_{t} E\left(\mathbf{e}_{j t} \mid s_{t}, \Omega_{t-1}\right)\right]=\mathbf{0}, \quad j=0,1 .
\end{aligned}
$$

Thus, the conditional expectation of $\mathbf{y}_{t}$, and one-period-ahead forecast, is a weighted average of the conditional expectations of the VAR regimes where the weight $p_{t}=E_{t-1}\left(s_{t}\right)$ is given in (5). All in all, in contrast to expressions (8) and (9), a simulation-based method is generally needed to obtain multiperiod forecasts (see Section 3.2).

\section{Estimation and forecasting}

\subsection{ML estimation}

The parameters of the QR-VAR model described in Section 2 can conveniently be estimated by the method of maximum likelihood (ML). The difficulties in the estimation of many previously considered (univariate and multivariate) regime switching models are typically related to the determination of the (unobserved) regimes and their conditional probabilities (see, e.g., Gray, 1996; Simpson et al., 2001; Ang and Bekaert, 2002a,b). In our approach, parameter estimation greatly simplifies because an observable binary time series determines the regime.

Conditional on the information set $\Omega_{t-1}$, the density function of $\boldsymbol{z}_{t}$ (see (1)) is 
characterized by

$$
g_{t-1}\left(\boldsymbol{z}_{t} ; \boldsymbol{\theta}\right)=f\left(\mathbf{y}_{t} \mid s_{t}, \Omega_{t-1} ; \boldsymbol{\theta}\right) P\left(s_{t} \mid \Omega_{t-1} ; \boldsymbol{\theta}\right),
$$

where $f\left(\mathbf{y}_{t} \mid s_{t}, \Omega_{t-1} ; \boldsymbol{\theta}\right)$ is the conditional density function of the random vector $\mathbf{y}_{t}$ conditional on the value of the binary variable $s_{t}$ and $P\left(s_{t} \mid \Omega_{t-1} ; \boldsymbol{\theta}\right)$ is the conditional probability mass function of $s_{t}$. The vector of parameters $\boldsymbol{\theta}$ contains all the parameters of the model. Assume that $\boldsymbol{\theta}=\left[\begin{array}{ll}\boldsymbol{\theta}_{1}^{\prime} & \boldsymbol{\theta}_{2}^{\prime}\end{array}\right]^{\prime}$, where $\boldsymbol{\theta}_{1}$ and $\boldsymbol{\theta}_{2}$ contain the parameters related to the regime switching VAR model (2) and to the model for the binary variable, respectively. The density function (10) can therefore be written as

$$
g_{t-1}\left(\boldsymbol{z}_{t} ; \boldsymbol{\theta}\right)=f\left(\mathbf{y}_{t} \mid s_{t}, \Omega_{t-1} ; \boldsymbol{\theta}_{1}\right) P\left(s_{t} \mid \Omega_{t-1} ; \boldsymbol{\theta}_{2}\right) .
$$

Under the normality assumption of $\mathbf{e}_{j t}, j=0,1$ (see (3)), the conditional density function of model (2) is

$$
f\left(\mathbf{y}_{t} \mid s_{t}, \Omega_{t-1} ; \boldsymbol{\theta}_{1}\right)=(2 \pi)^{-K / 2} \operatorname{det}\left(\boldsymbol{\Sigma}_{s_{t}}\right)^{-1 / 2} \exp \left(-\frac{1}{2} \mathbf{e}_{s_{t}, t}^{\prime} \boldsymbol{\Sigma}_{s_{t}}^{-1} \mathbf{e}_{s_{t}, t}\right), \quad s_{t}=0,1 .
$$

In the case of binary variable $s_{t}$, the conditional probability mass function is

$$
P\left(s_{t} \mid \Omega_{t-1} ; \boldsymbol{\theta}_{2}\right)=\left(\Phi\left(\pi_{t}\right)\right)^{s_{t}}\left(1-\Phi\left(\pi_{t}\right)\right)^{1-s_{t}}, \quad s_{t}=0,1,
$$

where $\pi_{t}$ is specified as in (6).

Assume that we have observed the time series $\mathbf{y}_{t}$ and $s_{t}, t=1,2, \ldots, T$, with the initial values treated as fixed constants. Based on the conditional density function (11) of $z_{t}$, the log-likelihood function over the whole sample, given the initial values, is

$$
l_{T}(\boldsymbol{\theta})=\sum_{t=1}^{T} l_{t}(\boldsymbol{\theta})=\sum_{t=1}^{T} \log f\left(\mathbf{y}_{t} \mid s_{t}, \Omega_{t-1} ; \boldsymbol{\theta}_{1}\right)+\sum_{t=1}^{T} \log P\left(s_{t} \mid \Omega_{t-1} ; \boldsymbol{\theta}_{2}\right),
$$

where the two factors of $g_{t-1}\left(\boldsymbol{z}_{t} ; \boldsymbol{\theta}\right)$ in (11) are defined in (12) and (13). Thus, $\boldsymbol{\theta}_{1}$ and $\boldsymbol{\theta}_{2}$ can be estimated separately and the maximum likelihood estimate $\widehat{\boldsymbol{\theta}}$ is 
obtained by maximizing (14) by numerical methods (see the models with a similar structure as (14), e.g., in Hamilton and Jordà (2002) and Nyberg (2012)).

\subsection{Computing multiperiod forecasts}

After an adequate description of the joint dynamics of the variables $s_{t}$ and $\mathbf{y}_{t}$ has been obtained, the QR-VAR model can be used to forecast the future values of the time series. An advantage of the QR-VAR model over the forecast horizon-specific binary response (see, e.g., Estrella and Mishkin, 1998; Kauppi and Saikkonen, 2008; Nyberg, 2010) and VAR models is that it leads to the dynamic iterative multiperiod forecasting approach (cf. the conventional VAR and the models of Dueker (2005) and Fornari and Lemke (2010)), without a need to specify a new model for every forecast horizon $h$ (i.e. the direct multiperiod forecasting approach).

As we concentrate on iterative multiperiod forecasting approach throughout this study, forecasts for the continuous dependent variables $\mathbf{y}_{t}$ are also needed to construct multiperiod forecasts for the binary variable $s_{t}$. Our model provides a simple and computationally feasible approach to obtain multiperiod forecasts of the variables included in $\mathbf{y}_{t}$, which is not the case for many regime switching models considered in the previous research with a dependence on the latent regimes and time-varying transition probabilities (cf. Section 3.1). In fact, in this multivariate case, to the best of our knowledge, multiperiod forecasting has not been considered when allowing for time-varying transition probabilities between the regimes, as in this study.

Based on the information set at time $T$, the optimal $h$-period-ahead forecast of $\boldsymbol{z}_{T+h}$ (in the mean-square sense) is the conditional expectation

$$
E_{T}\left(\boldsymbol{z}_{T+h}\right)=E\left(\boldsymbol{z}_{T+h} \mid \Omega_{T}\right)=\left[\begin{array}{ll}
E_{T}\left(s_{T+h}\right) & E_{T}\left(\mathbf{y}_{T+h}\right)
\end{array}\right]^{\prime},
$$

where the information set $\Omega_{T}$ includes the history of the time series $\boldsymbol{z}_{t}$ up to time $T$. Due to the recursive structure of the QR-VAR model, forecasts for the binary variable $s_{t}$ are constructed first. 
The one-period forecast of $s_{T+1}$ (cf. (5)) is given by

$$
p_{T+1}=E_{T}\left(s_{T+1}\right)=P_{T}\left(s_{T+1}=1\right)=\Phi\left(\pi_{T+1}\right) .
$$

In the case of model (6), the linear function $\pi_{T+1}=\nu+a \pi_{T}+\mathbf{y}_{T}^{\prime} \boldsymbol{b}$ depends only on the information available at time $T$ and, thus, the forecast (16) can be constructed straightforwardly. Following (8), the one-period forecast of $\mathbf{y}_{T+1}$ is the conditional expectation

$$
E_{T}\left(\mathbf{y}_{T+1}\right)=p_{T+1} \boldsymbol{\mu}_{1, T+1}+\left(1-p_{T+1}\right) \boldsymbol{\mu}_{0, T+1},
$$

where $\boldsymbol{\mu}_{j, T+1}=\boldsymbol{w}_{j}+\sum_{i=1}^{p_{j}} \boldsymbol{A}_{i, j} \mathbf{y}_{T-i+1}, j=0,1$, and $p_{T+1}$ is the one-period-ahead forecast of $s_{T+1}$ given in (16).

When the forecast horizon is longer than one period $(h>1)$, forecast computation becomes much more complicated. As an example, let us consider two-period forecasts $(h=2)$. As in $(16)$, the forecast of $s_{T+2}$ is the conditional expectation

$$
p_{T+2}=E_{T}\left(s_{T+2}\right)=P_{T}\left(s_{T+2}=1\right)=E_{T}\left(\Phi\left(\pi_{T+2}\right)\right),
$$

where following (7), we can write

$$
\begin{aligned}
\pi_{T+2} & =\nu+a \pi_{T+1}+\mathbf{y}_{T+1}^{\prime} \boldsymbol{b} \\
& =\nu+a^{2} \pi_{T}+a\left(\nu+\mathbf{y}_{T}^{\prime} \boldsymbol{b}\right)+\mathbf{y}_{T+1}^{\prime} \boldsymbol{b} .
\end{aligned}
$$

Thus, (18) depends nonlinearly, via the function $\Phi(\cdot)$, on the value $\mathbf{y}_{T+1}$ which is unknown at time $T$. In particular, the conditional expectation (18) is not, in general, equal to the conditional probability of outcome $s_{T+2}=1$ evaluated at the expected value of $\mathbf{y}_{T+1}$ given in (17). Decomposing $\mathbf{y}_{T+1}$ into an expected component $E_{T}\left(\mathbf{y}_{T+1}\right)$ and the innovation $\mathbf{y}_{T+1}-E_{T}\left(\mathbf{y}_{T+1}\right) \stackrel{\text { def }}{=} \mathbf{e}_{j, T+1}^{+}$, the conditional expectation (18) can be expressed as

$$
p_{T+2}=\int_{-\infty}^{\infty} \Phi\left(\nu+a^{2} \pi_{T}+a\left(\nu+\mathbf{y}_{T}^{\prime} \boldsymbol{b}\right)+\left(E_{T}\left(\mathbf{y}_{T+1}\right)+\mathbf{e}_{j, T+1}^{+}\right)^{\prime} \boldsymbol{b}\right) \varphi\left(\mathbf{e}_{j, T+1}^{+}\right) d \mathbf{e}_{j, T+1}^{+},
$$


where $\varphi\left(\mathbf{e}_{j, T+1}^{+}\right)$is the density function of $\mathbf{e}_{j, T+1}^{+}$. As this density function is intractable (especially at longer forecast horizons) and the integral above does not have a closed form solution, we cannot construct the forecast for $s_{T+2}$ using an explicit formula (cf. the one-period forecast (16)).

The two-period forecast of $\mathbf{y}_{T+2}$ can be expressed as

$$
\begin{aligned}
E_{T}\left(\mathbf{y}_{T+2}\right)= & E_{T}\left[s_{T+2}\left(\boldsymbol{w}_{1}+\boldsymbol{A}_{1,1} \mathbf{y}_{T+1}+\ldots+\boldsymbol{A}_{p_{1}, 1} \mathbf{y}_{T-p_{1}+2}+\mathbf{e}_{1, T+2}\right)+\right. \\
& \left.\left(1-s_{T+2}\right)\left(\boldsymbol{w}_{0}+\boldsymbol{A}_{1,0} \mathbf{y}_{T+1}+\ldots+\boldsymbol{A}_{p_{0}, 0} \mathbf{y}_{T-p_{0}+2}+\mathbf{e}_{0, T+2}\right)\right]
\end{aligned}
$$

In comparison to (17), as $E_{T}\left(s_{T+2} \mathbf{y}_{T+1}\right) \neq E_{T}\left(s_{T+2}\right) E_{T}\left(\mathbf{y}_{T+1}\right)$, we cannot take the conditional expectations of $s_{T+2}$ and the VAR regimes separately. The situation is similar when the forecast horizon $h$ lengthens. Thus, the expressions (18) and (19) demonstrate that there are no closed-form forecasting formulae (cf. the conventional VAR model) to construct multiperiod forecasts for $\mathbf{y}_{T+h}, h \geq 2$, and we have to resort to simulation-based forecasting techniques. The Monte Carlo forecasting procedure described below is, however, quite easy to implement and computationally feasible. It has some similarities to the forecasting methods employed for other (mainly univariate) nonlinear models (see, e.g., Teräsvirta et al., 2010, Chapter 14).

The essential idea is to simulate recursively a large number of independent realizations of the variables $s_{T+1}, \mathbf{y}_{T+1}, s_{T+2}, \mathbf{y}_{T+2}, \ldots$ Forecasts of $s_{T+h}$ and $\mathbf{y}_{T+h}$ for a given forecast horizon $h$ are then obtained as averages of the independently simulated realizations $s_{T+h}^{(i)}$ and $\mathbf{y}_{T+h}^{(i)}, i=1, \ldots, N$. The forecast horizon $h$ varies between 1 and $\bar{h}$ with $\bar{h}$ the maximum forecast horizon considered. Furthermore, for $h \geq 2$, let $\underline{\boldsymbol{z}}_{T+h-1}^{(i)}$ (cf. (1)) signify the vector containing the $i$ th simulated realizations $s_{T+1}^{(i)}, \mathbf{y}_{T+1}^{(i)}, \ldots, s_{T+h-1}^{(i)}, \mathbf{y}_{T+h-1}^{(i)}$ up to the forecast horizon $h-1$. Throughout it is assumed that the unknown values of the parameters, which in practice are replaced by their estimates, are known.

The forecast recursion for forecast horizons $h=1,2, \ldots, \bar{h}$ proceeds as follows: Step 1: Initialize $\pi_{T}^{(i)} \equiv \pi_{T}$ and $\mathbf{y}_{T-j}^{(i)} \equiv \mathbf{y}_{T-j}, j \geq 0$. Start the recursion with 
one-period forecast horizon i.e. set $h=1$ in Steps $2-5$.

Step 2: Compute $\left(\pi_{T+h}^{(i)} \mid \Omega_{T}, \underline{\boldsymbol{z}}_{T+h-1}^{(i)}\right)=\nu+a \pi_{T+h-1}^{(i)}+\mathbf{x}_{T+h-1}^{\prime(i)} \boldsymbol{b}$, where, e.g., if $K=2$ then $\mathbf{x}_{T+h-1}^{(i)}=\left[\begin{array}{ll}y_{1, T+h-k_{1}}^{(i)} & y_{2, T+h-k_{2}}^{(i)}\end{array}\right]^{\prime}$ for some $k_{1}$ and $k_{2} \geq 1$.

Step 3: Draw $\left(s_{T+h}^{(i)} \mid \Omega_{T}, \underline{\boldsymbol{z}}_{T+h-1}^{(i)}\right) \sim B\left(\Phi\left(\pi_{T+h}^{(i)}\right)\right)$, where $B(\cdot)$ denotes the Bernoulli distribution and $\pi_{T+h}^{(i)}$ is given in Step 2 (see (4)).

Step 4: Draw $\left(\mathbf{e}_{j, T+h}^{(i)} \mid s_{T+h}^{(i)}=j\right) \sim N\left(\mathbf{0}, \boldsymbol{\Sigma}_{j}\right), j=0,1$.

Step 5: Compute $\left(\mathbf{y}_{T+h}^{(i)} \mid \Omega_{T}, \underline{\boldsymbol{z}}_{T+h-1}^{(i)}, s_{t+h}^{(i)}=j\right)=s_{T+h}^{(i)}\left(\boldsymbol{w}_{1}+\boldsymbol{A}_{1,1} \mathbf{y}_{T+h-1}^{(i)}+\ldots\right.$ $\left.+\boldsymbol{A}_{p_{1}, 1} \mathbf{y}_{T+h-p_{1}}^{(i)}\right)+\left(1-s_{T+h}^{(i)}\right)\left(\boldsymbol{w}_{0}+\boldsymbol{A}_{1,0} \mathbf{y}_{T+h-1}^{(i)}+\ldots+\boldsymbol{A}_{p_{0}, 0} \mathbf{y}_{T+h-p_{0}}^{(i)}\right)+\mathbf{e}_{j, T+h}^{(i)}$.

Step 6: Go to Step 2 and repeat Steps $3-5$ starting from $h=2$ up to $h=\bar{h}$.

Step 7: Repeat Steps $2-6$ independently $N$ times $(i=1, \ldots, N)$.

The idea in the above recursion is first to use the horizon $h=1$ to obtain realizations $\pi_{T+1}^{(1)}, s_{T+1}^{(1)}, \mathbf{y}_{T+1}^{(1)}$. Next, the recursion is repeated for $h=2$, conditional on $\underline{\boldsymbol{z}}_{T+h-1}^{(i)}$, to obtain $\pi_{T+2}^{(1)}, s_{T+2}^{(1)}, \mathbf{y}_{T+2}^{(1)}$. This is continued up to $h=\bar{h}$. Finally, forecasts for $s_{T+h}$ and $\mathbf{y}_{T+h}, E_{T}\left(s_{T+h}\right)$ and $E_{T}\left(\mathbf{y}_{T+h}\right), h=1, \ldots, \bar{h}$, are obtained by computing the averages (cf. equation (15))

$$
\widehat{p}_{T+h}=\frac{1}{N} \sum_{i=1}^{N} s_{T+h}^{(i)}
$$

and

$$
\widehat{\mathbf{y}}_{T+h}=\frac{1}{N} \sum_{i=1}^{N} \mathbf{y}_{T+h}^{(i)},
$$

where $N$ is large. Note that the one-period forecasts $(h=1)$ obtained with (20) and (21) will be asymptotically equivalent to (16) and (17) but the above forecast recursion should accommodate also this horizon to start the recursion. In addition to point forecasts, the expressions (20) and (21) can straightforwardly be used to construct possibly asymmetric interval and density forecasts.

The accuracy of the proposed forecasting method depends on the choice of the number of replications $N$. For a good approximation, $N$ should be large enough. On the other hand, the larger the number of replications the more computationally burdensome the method is although simulation in Steps 3-4 is straightforward and not time consuming. The simulation results reported more detail in the Appendix 
suggest that the proposed method is accurate even for relative small values of $N$ (such as $N=10000$ ).

\section{Application: Forecasting U.S. interest rates and business cycle}

\subsection{Background and data set}

In our empirical application, we examine the bidirectional predictive linkages between the U.S. interest rates and the state of the business cycle measured in terms of recession and expansion periods. We are, in particular, interested in whether superior out-of-sample interest rate forecasts can be obtained with the proposed QR-VAR model over the single-regime VAR and alternative nonlinear VAR models, including the Markov switching and vector threshold VAR models.

We consider a monthly U.S. data set from January 1972 to December 2010. The starting point of the sample (i.e. the beginning of the 1970s) is consistent with many previous studies (see, e.g., Ang and Bekaert, 2002a,b; Huse, 2011). The state of the economy $s_{t}$ is determined by the National Bureau of Economic Research (NBER) business cycle turning points. That is in the binary time series $s_{t}$ the value $s_{t}=1$ indicates a recession and $s_{t}=0$ an expansion. The term spread $\left(T S_{t}\right)$ is the difference between the long-term (10-year government bond) and the short-term $i_{t}$ (three-month Treasury Bill rate) interest rates. The source of all data is the Federal Reserve Bank of St. Louis databank (FRED). Following the expectations hypothesis of the term structure of interest rates, the dynamics of the interest rates can be considered by using a bivariate model of $\mathbf{y}_{t}$ containing the term spread $\left(T S_{t}\right)$ and the first-difference of the short rate $\left(\Delta i_{t}\right)$ (see, e.g, Campbell and Shiller, 1991; Sola and Driffill, 1994). We are hence, for example, interested in knowing whether the term spread predicts the changes in the short rate (see, e.g., Ang and Bekeart, 2002a; Bansal et al., 2004) when the business cycle regime is taken into account. 
The main interest throughout this paper is in out-of-sample forecasts for the short-term interest rate. The short-term interest rate is of particular interest as it is a fundamental building block of many macroeconomic and financial models (see, e.g., the term structure (yield curve) models of Ang and Piazzesi (2003), Bansal et al. (2004), Diebold et al. (2006) and Huse (2011) incorporating macroeconomic variables or constructed factors). ${ }^{1}$ Here we propose an alternative to the examined econometric regime switching models for the short rate where the obtained regime probabilities for the latent regimes are often interpreted to describe regimes in real economic activity and compared with the NBER business cycle periods (see Filardo, 1994; Ang and Bekaert, 2002a).

Based on the structure of the QR-VAR model, the lags of $\mathbf{y}_{t}$ (i.e., the lags of the term spread and short rate) are used to predict the state of the business cycle $s_{t}$. Much of the previous research lends support, especially, to the term spread being a useful leading indicator of future real activity (see, e.g., Estrella and Mishkin, 1998; Estrella, 2005; Rudebusch and Williams, 2009). Ang et al. (2006) and Wright (2006) find that the short rate has also some additional predictive power.

Figure 1 lends support to the regime switching approach as the U.S. interest rate dynamics appears to be closely dependent on the state of the economy. The short rate has typically been increasing (decreasing) during the expansion (recession) periods while during the recessions (expansions) the term spread (yield curve) is generally upward (downward) sloping. All of the recession periods are preceded by a low, or even negative, value of the term spread, explaining why it has been found a useful leading indicator of the recession periods. Recession periods have also been characterized by a high short rate compared with its recent past just before the beginning of recession.

\footnotetext{
${ }^{1}$ In this study, we concentrate on the out-of-sample forecasting power of (nonlinear) econometric models. Yield curve (term structure) models provide an alternative class of models. Some attention has been paid on their out-of-sample forecasting ability, and so far the reported performances have often been rather disappointing (see Duffee (2002) and Diebold and Li (2006), and the references therein) compared with, e.g., the random walk examined in Section 4.4.
} 


\subsection{Estimation and model selection results}

In this section, we briefly discuss the estimation results of the QR-VAR model and examine the possible two-way linkage between the variables before proceeding to out-of-sample forecasting in Sections 4.3-4.4. A subsample period up to 1992:12 is used to select the models which are subsequently employed in out-of-sample forecasting for the period 1993:1-2010:12. Due to the recursive structure of the QR-VAR model, a model for the U.S. business cycle is specified first and treated independently of the regime switching VAR component (2).

Model selection results (available upon request) suggest that the third lag of the term spread $\left(T S_{t-3}\right)$ and the first lag of the differenced short rate $\left(\Delta i_{t-1}\right)$ are the best predictors of the state of the business cycle. The detailed estimation results of model (6) (based on the entire sample period), where $\mathbf{x}_{t-1}=\left[\begin{array}{lll}T S_{t-3} & \Delta i_{t-1}\end{array}\right]^{\prime}$, are presented in Table 1. Due to the negative and statistically significant coefficients, a low value of the term spread and decreasing short rate increase the probability of recession $\left(s_{t}=1\right)$. The values of the statistical goodness-of-fit measures, such as the pseudo- $R^{2}$ of Estrella (1998) and the area under the ROC curve (AUC) (see, e.g., Berge and Jordà (2011) and Lahiri and Yang (2013), and the references therein), and the probability of recession depicted in Figure 2 show that the selected model predicts the state of the U.S. business cycle accurately. The probability of recession is high during the recessions and close to zero in the expansion periods except for a few short exceptions. According to the test of Pesaran and Timmermann (2009) allowing for serial correlation in $s_{t}$, the model is able to predict the state of the business cycle at the $5 \%$ significance level.

Overall, the model matches the U.S. business cycle regimes accurately which has not always been the case in the previous regime switching models when aiming to obtain a correspondence with the NBER business cycle periods. ${ }^{2}$ In fact, the obtained transition probabilities for the unobserved regimes have not been found

\footnotetext{
${ }^{2}$ Of course the regimes can also be dictated by some other factors than real activity such as the stance of monetary policy (see, e.g., Sims and Zha, 2006; Bikbov and Chernov, 2013), but in this study we link the regimes to the NBER business cycles.
} 
to necessarily describe business cycle recession and expansion periods. Instead, Filardo (1994) and Henkel et al. (2011), among others, interpret the transition probabilities to describe low and high growth rate regimes in the real GDP which describe more general contraction and expansion periods in real activity than business cycles which are due to the structure of the model explicitly used in our analysis.

Next we turn our interest to the estimation results of the regime switching VAR model (2). So far, we have assumed that the lag lengths $p_{0}$ and $p_{1}$ in the $\mathrm{QR}-\operatorname{VAR}\left(p_{0}, p_{1}\right)$ model are known. In the previous research, Ang and Bekaert (2002a,b) and Henkel et al. (2011), among others, have restricted themselves to the parsimonious first-order regime switching VAR models $\left(p_{0}=p_{1}=1\right)$. This is also a reasonable benchmark in this study. According to our estimation sample period 1972:1-1992:12, the Bayesian information criterion favors the QR-VAR(1,1) and linear VAR(3) models while the Akaike criterion suggests the maximum sixthorder models. A sequential testing procedure, where the Likelihood ratio (LR) test is applied sequentially when the order of the model increases until the first nonrejection, selects the QR-VAR $(4,3)$ and $\operatorname{VAR}(3)$ models. Irrespective of the selected QR-VAR or VAR models, there is some evidence of remaining autocorrelation in the equation of the short rate and conditional heteroskedasticity in both variables, but among the examined specifications, the QR-VAR(4,3) model seems the best selection also in terms of the diagnostic checks.

In Table 2, we illustrate, for simplicity, the estimation results of the parsimonious QR-VAR(1,1) and VAR(1) models. Above the model selection was carried out using the sample period 1972:1-1992:12, but the estimation results in Table 2 are, for illustrative purposes, presented for the full sample period 1972:1-2010:12 to include more recession periods to the sample. The results of the QR-VAR(4,3) and $\operatorname{VAR}(3)$ models are available upon request. In the QR-VAR $(1,1)$ model, the parameter estimates, especially the constant terms, are different across the business cycle regimes and from the ones of the VAR(1) model. In line with Figure 1, the constant term for the first-difference of the short rate is negative in the 
recession regime. The term spread is a useful predictor of the short rate mainly in the recession regime. Interestingly, the persistence in the term spread appears much higher in the expansion regime. Furthermore, the higher standard errors of estimated parameter coefficients in the recession regime are most likely resulting from the small number of observations in the recession regime.

Overall, irrespective of the lag length selection (results not reported), the QRVAR model outperforms the VAR model as we can strongly reject the hypothesis of equal parameter coefficients in the expansion and recession regimes at all traditional significance levels. Thus, there appears to exist a bidirectional in-sample predictive linkage between the variables: The lags of the term spread and short rate predict the state of the business cycle (see Table 1). On the other hand, the VAR dynamics are strongly dependent on the business cycle regime (see Table 2). The estimated covariance matrices $\boldsymbol{\Sigma}_{0}$ and $\boldsymbol{\Sigma}_{1}$ are also different in two business cycle regimes. In particular, the diagonal elements are clearly higher in the recession regime implying higher volatility.

\subsection{Out-of-sample forecasting}

We compare the (real-time) out-of-sample forecasts obtained with the proposed QR-VAR model and various alternative linear and regime switching VAR models for the period 1993:1-2010:12. The main interest is in interest rate forecasts (i.e. the variables included in the VAR). We concentrate first on the comparison between the QR-VAR and linear VAR models to examine the effect of allowing for business cycle-specific regimes in the VAR model. Later on in Section 4.4, we will also compare the forecasting performance with, e.g., the Markov switching model.

Forecasts are computed using an expanding window approach where the estimation sample period increases in each time when the parameters are re-estimated until the end of the sample. Based on the Monte Carlo forecasting experiments presented in the Appendix, the number of simulated realizations $N$ in the simulationbased forecasting method is fixed to 10000 . 
Using the NBER recession dates naturally raises concerns on the real time implementability of the QR-VAR model in forecasting as the business cycle turning points determining the values of $s_{t}$ are not available in real time. This is, however, taken into account in various ways. First of all, as a part of the model we forecast the values of $s_{t}$ using only the information available in real time (see Steps 2 and 3 in Section 3.2). Second, we do not employ the lags of $s_{t}$ in model (6) circumventing complications related to their use as predictors (cf. the models and discussion in Kauppi and Saikkonen (2008) and Nyberg (2010)). Third, parameters are reestimated only when a complete business cycle from trough month to the next trough has been completed (i.e. the business cycle trough point is identified in real time) to facilitate a fair comparison between the models. Therefore, the outof-sample forecasting period starts after the announcement of the business cycle trough for March 1991 made by the NBER in December 1992.

In Table 3, following the previous literature on the regime switching VAR models, we report the results of the first-order QR-VAR(1,1) model along with the QR-VAR(4,3) model. The relative MSFE and QPS statistics are obtained relative to the single-regime $\operatorname{VAR}(1)$ and $\operatorname{VAR}(4)$ models and the univariate autoregressive probit (6) model. The VAR(4) model is used as a single-regime counterpart of the QR-VAR(4,3) model instead of the VAR(3) model (suggested by the BIC and sequential model selection procedure) as the $\operatorname{VAR}(3)$ (results available upon request) leads to inferior out-of-sample forecast performance compared with the VAR(4). The forecast evaluation for the short rate is executed for its level which is of interest in many applications and can easily be computed from the forecasts of the first-difference of $i_{t}$. Under the hypothesis of no business cycle-specific regimes the QR-VAR model nests the VAR model as a special case. Thus, the test of Clark and West (2007) is used to test the equal predictive performance between the QR-VAR and VAR models. The QR-VAR and univariate forecast horizonspecific models for the binary variable are not (generally) nested and, thus, the Diebold-Mariano (1995) and West (1996) test is employed in that case.

Many interesting findings emerge. Let us first consider forecasts for the short 
rate which are of most interest in this analysis. It can be seen that the QR-VAR $(1,1)$ and QR-VAR(4,3) models clearly outperform their corresponding single-regime $\operatorname{VAR}(1)$ and $\operatorname{VAR}(4)$ models. Depending on the forecast horizon, the relative differences in the forecast accuracy typically range from $5 \%$ to even $20 \%$. The firstorder (QR-VAR(1,1)) model seems to yield better forecasts than the QR-VAR(4,3) model. Based on the test of Clark and West (2007), the differences between the QR-VAR and VAR models are statistically significant at all the conventional significance levels showing the superior predictive performance of the former model.

The results for the term spread are basically the same as for the short rate. In this case, the QR-VAR $(4,3)$ model produces somewhat better forecasts than the QR-VAR $(1,1)$ model. However, in both cases, the QR-VAR models outperform the VAR models by a clear margin. The relative MSFEs are throughout below unity and the $p$-values of the Clark and West (2007) test are essentially zero.

As in Kauppi and Saikkonen (2008) and Nyberg (2010), the univariate autoregressive probit model (6) yields good forecasts for the state of the U.S. business cycle when the forecast horizon is relatively short. However, as expected and consistent with the simulation forecasting results presented in the Appendix, when the forecast horizon lengthens towards the maximum 12-month horizon, the dynamic iterative forecasting approach employed in the QR-VAR model outperforms the forecast horizon-specific univariate model. According to the Diebold-Mariano and West test the differences are not, however, statistically significant. All in all, in possible future applications, such as impulse response analysis within the QRVAR model (cf. Dueker, 2005, Fornari and Lemke, 2010), the dynamic iterative forecasting approach proposed in this study seems more appropriate.

It is also worth noting that the linear VAR can, in principle, be estimated recursively using real-time data observed at each time $t$. Due to the dependence on business cycle regimes and thus the publication lag in the values of $s_{t}$, this is not the case in the QR-VAR model in real time. This can be seen as a drawback for the QR-VAR model. Hence, another comparison between models could be performed by allowing for all the non-QR-VAR models to be estimated using 
the entire information set available. How substantial is this drawback? In fact, the forecasting performance of the VAR model estimated at each step yields slightly inferior forecasts than the one reported in Table 3 (details available upon request). Hence the main message of this and the next section, the importance of taking the predictable regimes into account in forecast construction, remains intact.

To get more detailed information where the forecasting gains are coming, in Table 4 we report the relative MSFEs separately for the business cycle expansions and recessions (Panel A). For the short rate the forecasting gains are larger in expansions but also at recession periods the QR-VAR model outperforms the linear VAR. For the term spread the forecasting gains are coming from expansions.

In Panel B of Table 4, the Area Under the ROC Curve (AUC) summarizes the out-of-sample predictive power obtained for the state of the U.S. business cycle. As in in-sample estimation results (Table 1), following the testing procedure used, for example, in Berge and Jordà (2011), the reported AUCs are all statistically significantly higher than 0.5 at the conventional significance levels. ${ }^{3}$ In other words, the proposed QR-VAR model is able to predict U.S. business cycles out of sample, providing the necessary ingredient to obtain superior predictive power also for interest rate variables. It appears that the only notable exception is approximately the period between the years 1998-1999 where the probability of recession is falsely somewhat high early in advance the recession started in 2001.

As a by-product of the simulation-based forecasts in the proposed QR-VAR model, we also obtain generally asymmetric density and interval forecasts. A more extensive examination is left for the future research but some general remarks can already be made. As expected, the interval forecasts (predictive densities) are generally somewhat wider when allowing for business cycle regimes in the VAR (see, e.g., the differences in the diagonal elements of the covariance matrices reported in Table 2). Due to wider forecast intervals, especially during the uncertain times

\footnotetext{
${ }^{3}$ The (out-of-sample) AUC gets values between 0 and 1, with the values of 0.5 and 1 corresponding a coin toss and perfect forecasts, respectively. In a growing number of economic applications (see, e.g., Berge and Jordà, 2011; Lahiri and Yang, 2013; Berge, 2015; Nyberg and Pönkä, 2016), the AUC has been used as a statistical goodness-of-fit measure for binary time series.
} 
around business cycle turning points, the QR-VAR model contains the observed short rate more often than the linear VAR model.

As a whole, we can conclude that superior forecasts for the interest rate variables can be obtained by allowing for the business cycle-specific regimes and, in particular, utilizing the predictability of those regimes in forecasting. In the previous studies, the relative differences between the single-regime and regime switching models have typically been smaller than in this study (see, e.g., Filardo, 1994, Ang and Bekaert, 2002a). In this respect, the proposed model turns out to perform really well. Dacco and Satchell (1999) show that a regime-switching model, based on the latent regimes, may have poor forecasting performance relative to a linear model as a result of misclassifying observations to wrong regimes. Clements et al. (2004) have also emphasized that the relative performance of the regime switching model is expected to improve when the regimes are persistent. The fact the QR-VAR model is based on the observable and persistent NBER business cycle regimes helps in parameter estimation which seems to lead subsequent forecasting gains. This interpretation is still examined more detail in the next section when comparing the forecasting performance with, e.g., the Markov switching model.

\subsection{Additional out-of-sample forecasting checks}

Instead of the bivariate linear VAR and QR-VAR models, in this section we consider several additional out-of-sample forecasting checks for the findings obtained in Section 4.3. In particular, we examine augmented (three-variable) VAR and QR-VAR models as well as the predictive performance of the random walk, which has often been found to yield superior forecasts over, for example, various yield curve models (see, e.g., Duffee, 2002). In addition, we assess the value added of the QR-VAR model when comparing its out-of-sample forecasting performance with the Markov switching VAR and vector threshold VAR models (which is also interpreted as a regime switching model in this study).

An alternative approach to the QR-VAR model to explore the relationship 
between the interest rates and business cycle can be based on an augmented linear VAR model by simply augmenting $\mathbf{y}_{t}$ (including interest rate variables) by a continuous variable measuring real economic activity. In other words, instead of using the binary variable determining the business cycle regime, we can specify a three-variable VAR model where in addition to the term spread $\left(T S_{t}\right)$ and the first-difference of the short rate $\left(\Delta i_{t}\right)$ a growth rate of industrial production (ipt) or nonfarm payroll employment (emp) is also included in the model. Ultimately, the comparison between this traditional and the QR-VAR-based regime switching approach is about what is the best way of forecasting interest rates and real activity (business cycles) and their linkages.

Industrial production and employment have probably been the most commonly used monthly indicators of real activity. Because of real-time data availability issues, we use the real-time data of industrial production and employment available at the Federal Reserve Bank of Philadelphia website. In addition to data revisions, we are also taking the one-month information lag of both variables into account in out-of-sample forecasting (cf. the informational lag in the NBER business cycle turning points). Therefore, nowcasts for their values at time $T$ are constructed first with the same models which are used to compute forecasts for the future.

Similarly as in Table 3, we report the MSFEs (QPSs for the binary variable) of different models in Table 5. Following the results obtained in Table 3, parsimonious models seem to overall produce slightly superior forecasts than the models with longer lag lengths. Therefore, the models presented in Table 5 are selected based on the BIC including the QR-VAR $(1,1)$ model (which out-of-sample forecasting performance is already presented in Table 3$)$. It turns out that the QR-VAR(1,1) model is generally the best model also in this comparison, especially for the shortterm interest rate. In particular, it outperforms the three-variable VAR models including industrial production or employment as a third variable. In other words, linking interest rates to real activity in a nonlinear (business cycle-specific) regime switching fashion leads to superior out-of-sample forecasts compared with the augmented linear models. Furthermore, the examined QR-VAR models also outper- 
form the random walk (by a wide margin) irrespective of the forecast horizon for both variables (term spread and short rate).

For the term spread, the results are essentially similar as for the short rate. The only exception is the three-variable $\operatorname{VAR}(2)$ model including employment $(\operatorname{VAR}(2)+\mathrm{emp})$. However, it is worth noting that its forecasting performance for the short rate is clearly inferior compared with the QR-VAR models. It appears that including the third continuous variable in the QR-VAR model (in the vectors $\mathbf{y}_{t}$ and $\mathbf{x}_{t-1}$ ) does not improve interest rate forecasts and does not have a substantial effect on the forecasts for the state of the business cycle $\left(s_{t}\right)$ even though for some forecast horizons the QPS statistics are slightly smaller than in the best QR-VAR $(1,1)$ model presented in Table 3.

As discussed in the Introduction, the QR-VAR model can be seen as an alternative to the Markov switching VAR (MSVAR) model. Time-varying regime probabilities obtained with the QR-VAR model would thus ideally lead to a comparison between the MSVAR model with time-varying transition probabilities. However, to the best of our knowledge, in the previous univariate and multivariate regime switching models with dependence on latent regimes and time-varying transition probabilities (based on the lagged values of $\mathbf{y}_{t}$, as in model (6)), including MSVAR models, only one-period-ahead forecasts (forecasting methods) have been considered so far (see, e.g., Filardo, 1994; Perez-Quiros and Timmermann, 2000; Simpson et al., 2001; Ang and Bekaert, 2002a). Because we are especially interested in multiperiod forecasting, this previous work is thus not directly comparable to ours. As the multistep forecasting procedures and their properties are unknown at the moment, we use the MSVAR model with fixed transition probabilities where the multiperiod forecasts can be obtained using analytical expressions (see details, e.g., in Teräsvirta et al., 2010, pp. 346-347).

In Table 6, we compare the out-of-sample forecasting performance of the QRVAR model to the MSVAR model with constant transition probabilities to get evidence whether it is advantageous in terms of interest rate forecasts (variables included in the VAR) to relate the regimes to the NBER periods compared with 
latent regime approach. In the MSVAR model, the extracted regimes maximize the statistical likelihood of a switching model which can be more relevant to the interest rate variables and dictated by other economic forces than the business cycles such as the stance of the monetary policy (see, e.g., Sims and Zha, 2006; Bibkov and Chernov, 2013). ${ }^{4}$

Another forecasting comparison check is made with a vector threshold VAR (VTVAR) model (see Teräsvirta et al., 2010, pp. 34-35). In the VTVAR model, we assume that the first lag of the term spread is used as the threshold variable for both VAR equations. For example, when the full sample period is used in estimation, the threshold value between the regimes is 0.13 (cf. the right panel of Figure 1). As above in Table 5, the results of the VTVAR and MSVAR models are based on the models where the lag lengths are selected using the BIC.

Table 6 reports the relative MSFEs between the QR-VAR model and MSVAR and VTVAR models, respectively. The entries below unity show the superiority of the QR-VAR model. Overall, it turns out that the QR-VAR $(1,1)$ model (selected based on the BIC as well) produces superior out-of-sample forecasts over the competing models. According to the Diebold-Mariano and West test, the differences in the forecast accuracy are also statistically significant at least at the $10 \%$, in many cases even at the $5 \%$, significance levels. This is, especially, the case when the forecast horizon lengthens.

\section{Conclusions}

Regime switching models provide an attractive class of econometric models to capture regime changes in the stochastic behavior of interest rates. In this study, we suggest a new regime switching VAR model, referred for simplicity to as the QR-VAR model, which can also be seen as a joint model between real-valued continuous and qualitative dependent variables. The model is easier to work with and

\footnotetext{
${ }^{4}$ In the case of the Markov switching model, we employ the MS Regress package for Matlab (see Perlin 2012: MS Regress - The MATLAB Package for Markov Regime Switching Models (http://ssrn.com/abstract=1714016)).
} 
interpret than some previously considered multivariate regime switching models where the latent regimes are determined within the econometric model. Although a simulation-based forecasting method is required to construct multiperiod forecasts, the proposed method is not computationally burdensome.

The QR-VAR model is applied to forecast the U.S. interest rates and the state of the business cycle. The empirical results show that there is a strong bidirectional linkage between the U.S. business cycle measured in terms of the NBER expansion and recession periods and the bivariate system of the U.S. term spread and the changes in the short-term interest rate. The results can be interpreted as positive evidence for a reduced-form model for the short rate incorporating business cycle shifts as the term spread and the short rate help to predict the future business cycle regimes while the state of the business cycle has also feedback effects back to them. Most importantly, the ability of the QR-VAR model to forecast business cycle turning points leads to superior out-of-sample forecasting performance for the interest rate variables compared with the conventional single-regime VAR model and previously considered nonlinear VAR models, including the Markov switching VAR model.

The QR-VAR model can be extended various ways. One possibility is to replace the binary variable with other qualitative response variable, such as a multinomial variable allowing for more than two regimes. Another interesting extension could be to use the model in structural macroeconomic analysis where the impulse response functions implied by the QR-VAR model, with alternating regimes, may lead to different conclusions than the VAR or other regime switching models employed in the previous literature. To facilitate impulse response analysis, forecasts for the future values of the variables are required and, therefore, the proposed simulationbased iterative forecasting method, and subsequent results, are also of interest. Examining the interval and density forecasting performance of the proposed model more detail in different applications might also be a worthwhile extension to this study. 


\section{Appendix: Monte Carlo forecasting experiment}

As discussed in Section 3.2, a simulation-based forecasting procedure is generally required to construct multiperiod forecasts in the QR-VAR model. In the proposed MC simulation method, the essential task is to specify the number of simulation replications $N$ that affects the approximation error coming from the numerical integration. Thus, we consider a small-scale Monte Carlo simulation experiment in order to specify the number of replications $N$ and illustrate the properties and the usefulness of the forecasting method. The data generating process (DGP) is based on the QR-VAR $(1,1)$ model presented in Tables 1-2.

We simulate 5000 realizations of length $T+12$ observations from the abovementioned DGP. Using the first $T$ observations in each realization, we estimate the univariate probit model (6) and the VAR model along with the true QR-VAR model. Forecasts are computed for the forecast horizons from 1 to 12 periods. The mean-squared forecast errors (MSFE) and the QPS statistics (Diebold and Rudebusch, 1989) for the continuous and binary dependent variables are constructed, respectively. We experiment with two sample sizes $(T=200$ and $T=500)$ and three choices of $N(1000,10000$ and 50000$)$.

Table 7 presents the MSFE and QPS statistics of the QR-VAR model for different forecast horizons. The accuracy of forecasts for the binary variable appears to increase with the sample size $T$ while this effect is not so clear for the continuous variables. As far as the number of replications is concerned, there is a slight improvement when $N$ increases from 1000 to 10 000, but basically no changes when $N$ increases from 10000 to 50000 . Thus, in conclusion, $N=10000$ appears to be a sufficient selection.

The relative MSFE and QPS statistics in Table 8 are obtained by dividing the MSFE and the QPS statistics of the QR-VAR model reported in Table 7 by those of the corresponding $\operatorname{VAR}(1)$ and univariate probit (6) models. Most of entries are below unity for the variables $y_{1 t}$ and $y_{2 t}$ indicating the superiority of the true QR-VAR specification over the VAR model. The relative MSFEs in Table 7 are 
essentially the same with different selections of $N$. The relative QPS statistics for the binary variable show that the QR-VAR model designed to construct dynamic iterative multiperiod forecasts outperforms the forecast horizon-specific univariate model when the forecast horizon lengthens. As pointed out in Section 3.2, the one-period forecasts from the QR-VAR and the univariate autoregressive probit models are asymptotically equal.

\section{Acknowledgements}

The author would like to thank the Editor, anonymous referee, as well as Graham Elliott, Markku Lanne, Pentti Saikkonen and the participants at the 7th Nordic Econometric Meeting (Bergen, 2013), the 7th International Conference on Computational and Financial Econometrics (London, 2013), the 34th International Symposium on Forecasting (Rotterdam, 2014) and the 8th conference on Growth and Business Cycles in Theory and Practice (Manchester, 2016) for useful comments. Financial support from the Academy of Finland and the Research Funds of the University of Helsinki is gratefully acknowledged. Part of this research was done during the author's visit at the Faculty of Economics of the University of Cambridge (2011-2012) whose hospitality is also gratefully acknowledged. A previous discussion paper version of this study was distributed under the heading "A Qualitative Response VAR Model: An Application to Joint Dynamics of U.S. Interest Rates and Business Cycle".

\section{References}

Ang, A., Bekaert, G. (2002a). Regime switches in interest rates. Journal of Business and Economic Statistics, 20, 163-182.

Ang, A., Bekaert, G. (2002b). Short rate nonlinearities and regime switches. Journal of Economic Dynamics and Control, 26, 1243-1274.

Ang, A., Piazzesi, M. (2003). A no-arbitrage vector autoregression of term 
structure dynamics with macroeconomic and latent variables. Journal of Monetary Economics, 50, 745-787.

Ang, A., Piazzesi, M., Wei, M. (2006). What does the yield curve tell us about the GDP growth? Journal of Econometrics, 131, 359-403.

Bansal, R., Tauchen, G., Zhou, H. (2004). Regime shifts, risk premiums in the term structure, and the business cycle. Journal of Business and Economic Statistics, 22, 396-409.

Benjamin, M.A., Rigby, R.A., Stasinopoulos, D.M. (2003). Generalized autoregressive moving average models. Journal of American Statistical Association, 98, $214-223$.

Berge, T.J. (2015). Predicting recessions with leading indicators: Model averaging and selection over the business cycle. Journal of Forecasting 34, 455-471.

Berge, T.J., and O. Jordà (2011). Evaluating the classification of economic activity into recessions and expansions. American Economic Journal: Macroeconomics 3, 246-277.

Bikbov, R., Chernov, M. (2013). Monetary policy regimes and the term structure of interest rates. Journal of Econometrics, 174, 27-43.

Campbell, J.Y., Shiller, R.J. (1991). Yield spreads and interest rate movements: A bird's eye view. Review of Economic Studies, 58, 495-514.

Clark, T.E., West, K.D. (2007). Approximately normal tests for equal predictive accuracy in nested models. Journal of Econometrics, 138, 291-311.

Clements, M.P., Franses, P.H., Swanson, N.R. (2004). Forecasting economic and financial time series with non-linear models. International Journal of Forecasting, $20,169-183$.

Dacco, R., Satchell, S. (1999). Why do regime-switching models forecast so badly? Journal of Forecasting, 18, 1-16.

Diebold, F.X., Mariano, R.S. (1995). Comparing predictive accuracy. Journal of Business and Economic Statistics, 13, 134-144.

Diebold, F.X., Rudebusch, G.D. (1989). Scoring the leading indicators. Journal of Business, 62, 369-391. 
Diebold, F.X., Li, C. ((2006). Forecasting the term structure of government bond yields. Journal of Econometrics, 130, 337-364.

Diebold, F.X., Rudebusch, G.D., Aruoba, S.B. (2006). The macroeconomy and the yield curve: A dynamic latent factor approach. Journal of Econometrics, 131, $309-338$.

Dueker, M. (2005). Dynamic forecasts of qualitative variables: A Qual VAR model of U.S. recessions. Journal of Business and Economic Statistics, 23, 96-104.

Dueker, M.J., Psaradakis, Z., Sola, M., Spagnolo, F. (2011). Multivariate contemporaneous-threshold autoregressive models. Journal of Econometrics 160, 311-325.

Duffee, G. (2002). Term premia and interest rate forecasts in affine models. Journal of Finance, 57, 405-443.

Estrella, A. (1998). A new measure of fit for equations with dichotomous dependent variables. Journal of Business and Economic Statistics, 16, 198-205.

Estrella, A. (2005). Why does the yield curve predict output and inflation? Economic Journal, 115, 722-744.

Estrella, A., Mishkin, F.S., (1998). Predicting U.S. recessions: Financial variables as leading indicators. Review of Economics and Statistics, 80, 45-61.

Filardo, A. (1994). Business-cycle phases and their transitional dynamics. Journal of Business and Economic Statistics, 12, 299-308.

Filardo, A., Gordon, S.F. (1998). Business cycle durations. Journal of Econometrics, 85, 99-123.

Fornari, F., Lemke, W. (2010). Predicting recession probabilities with financial variables over multiple horizons. European Central Bank, Working Paper Series, No. 1255.

Gray, S. (1996). Modeling the conditional distribution of interest rates as a regime-switching process. Journal of Financial Economics, 2, 211-250.

Guidolin, M., Timmermann, A. (2006). An econometric model of nonlinear dynamics in the joint distribution of stock and bond returns. Journal of Applied Econometrics, 21, 1-22.

Hamilton, J.D., Jordà, O. (2002). A model of the Federal Funds rate target. 
Journal of Political Economy, 110, 1135-1167.

Henkel, S.J., Martin, J.S., Nardari, F. (2011). Time-varying short-horizon predictability. Journal of Financial Economics, 99, 560-580.

Huse, C. (2011). Term structure modelling with observable state variables. Journal of Banking and Finance, 35, 3240-3252.

Kauppi, H., Saikkonen, P. (2008). Predicting U.S. recessions with dynamic binary response models. Review of Economics and Statistics, 90, 777-791.

Krolzig, H.M. (1997). Markov-Switching Vector Autoregressions: Modelling, Statistical Inference, and Application to Business Cycle Analysis. Springer, Berlin.

Lahiri, K., Yang, L. (2013). Forecasting binary outcomes. In G. Elliott and A. Timmermann (eds.) Handbook of Economic Forecasting, Vol. 2B (1025 - 1106), Elsevier, North-Holland.

Nyberg, H. (2010). Dynamic probit models and financial variables in recession forecasting. Journal of Forecasting, 29, 215-230.

Nyberg, H. (2012). Risk-return tradeoff in U.S. stock returns over the business cycle. Journal of Financial and Quantitative Analysis, 47, 137-158.

Nyberg, H., Pönkä, H. (2016). International sign predictability of stock returns: The role of the United States. Forthcoming in the Economic Modelling.

Perez-Quiros, G., Timmermann, A. (2000). Firm size and cyclical variations in stock returns. Journal of Finance, 55, 1229-1262.

Pesaran, H.M., Timmermann, A. (2009). Testing dependence among serially correlated multicategory variables. Journal of the American Statistical Association, 104, 325-337.

Rudebusch, G.D., Williams, J.C. (2009). Forecasting recessions: The puzzle of the enduring power of the yield curve. Journal of Business and Economic Statistics, 27, 492-503.

Rydberg, T., Shephard, N. (2003). Dynamics of trade-by-trade price movements: Decomposition and models. Journal of Financial Econometrics, 1, 2-25.

Sensier, M., M. Artis, D.R. Osborn, Birchenhall, C. (2004). Domestic and international influences on business cycle regimes in Europe. International Journal 
of Forecasting, 20, 343-357.

Simpson, P.W., Osborn, D.R, Sensier, M. (2001). Forecasting UK industrial production over the business cycle. Journal of Forecasting, 20, 405-424.

Sims, C., Zha, T. (2006). Were there regime switches in U.S. monetary policy? American Economic Review, 96, 54-81.

Sola, M., Driffill, J. (1994). Testing the term structure of interest rates using a stationary vector autoregression with regime switching. Journal of Economic Dynamics and Control, 18, 601-628.

Teräsvirta, T., Tjøstheim, D., Granger, C. (2010). Modelling Nonlinear Economic Time Series. Oxford University Press, Oxford.

West, K.D. (1996). Asymptotic inference about predictive ability. Econometrica 64, 1067-1084.

Wright, J. (2006). The yield curve and predicting recessions. Finance and Economics Discussion Series No. 2006-07, Federal Reserve Board of Governors. 


\section{Tables and Figures}

Table 1: Estimation results of the autoregressive binary response model (6).

\begin{tabular}{ccccc}
\hline \hline$\pi_{t}$ & $\nu$ & $a$ & $b_{1}$ & $b_{2}$ \\
& & $\left(\pi_{t-1}\right)$ & $\left(T S_{t-3}\right)$ & $\left(\Delta i_{t-1}\right)$ \\
& 0.066 & 0.935 & -0.119 & -0.319 \\
& $(0.014)$ & $(0.009)$ & $(0.015)$ & $(0.074)$ \\
\hline $\mathrm{psR}^{2}$ & 0.419 & & $\mathrm{QPS}$ & 0.152 \\
$\mathrm{CR}_{50 \%}$ & 0.893 & & $\mathrm{CR}_{25 \%}$ & 0.849 \\
$\mathrm{PT}_{50 \%}$ & $6.887(0.009)$ & & $\mathrm{PT}_{25 \%}$ & $5.408(0.020)$ \\
$\mathrm{AUC}$ & 0.931 & & &
\end{tabular}

Notes: In the table, $\overline{\overline{T S_{t-3}} \text { and } \Delta i_{t-1} \text { denote the third and first lags of the term spread and the first }}$ difference of the short rate, respectively, included in $\mathbf{x}_{t-1}$ as predictors. The estimated coefficients are based on the full sample period (1972:1-2010:12) and their standard errors, based on the Hessian of the log-likelihood function, are given in the parentheses. The pseudo- $R^{2}$ of Estrella (1998) (psR $\left.{ }^{2}\right)$ and the QPS statistic (Diebold and Rudebusch, 1989) are the counterparts of the coefficient of determination and the mean-square prediction error used in linear models. $\mathrm{CR}_{50 \%}$ and $\mathrm{CR}_{25 \%}$ denote the percentages of correct recession and expansion signal forecasts when the $50 \%$ and $25 \%$ thresholds are used to construct signal forecasts from the probability of recession (see (5)). PT denotes the Pesaran-Timmermann (2009) test statistics ( $p$-values in the parentheses) for the null hypothesis that the state of the business cycle $\left(s_{t}\right)$ is unpredictable. AUC is the area under the Receiver Operating characteristic Curve (ROC) used to evaluate the classification ability of the model (see, e.g., Berge and Jordà, 2011, and Lahiri and Yang, 2013, and the references therein). 
Table 2: Estimation results of the QR-VAR(1,1) and VAR(1) models.

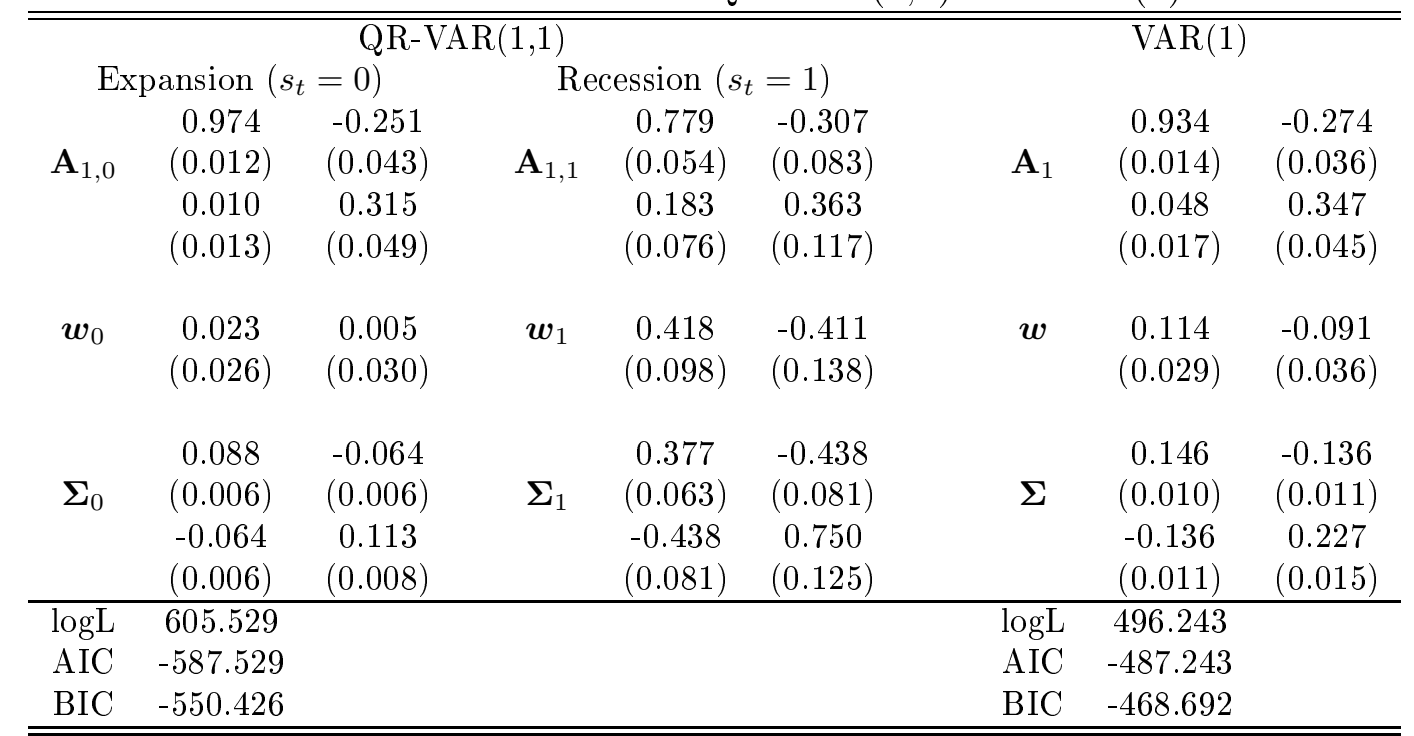

Notes: Estimation results are for the bivariate system including the term spread and the first-difference of the short-term interest rate (i.e. $\mathbf{y}_{t}=\left[\begin{array}{lll}T S_{t} & \Delta i_{t}\end{array}\right]^{\prime}$, the observations at period $t$ ). In the QR-VAR model, the reported values of the $\log$-likelihood function $(\log \mathrm{L})$ and the Akaike and Schwarz information criteria (AIC and BIC) are based only on the VAR part of the model. In this table, the full sample period (1972:1-2010:12) is used to estimate the parameters. 
Table 3: Out-of-sample forecasting performance of the QR-VAR and VAR models.

\begin{tabular}{|c|c|c|c|c|c|c|}
\hline \multirow[t]{2}{*}{ Model } & \multicolumn{6}{|c|}{ Forecast horizon (months) } \\
\hline & 1 & 2 & 3 & 6 & 9 & 12 \\
\hline \multicolumn{7}{|c|}{ MSFE, term spread $\left(T S_{t}\right)$} \\
\hline QR-VAR $(1,1)$ & 0.059 & 0.151 & 0.239 & 0.515 & 0.761 & 0.982 \\
\hline $\operatorname{VAR}(1)$ & 0.062 & 0.166 & 0.269 & 0.623 & 0.929 & 1.158 \\
\hline relative MSFE & $0.944^{* * *}$ & $0.909^{* * *}$ & $0.868^{* * *}$ & $0.826^{* * *}$ & $0.819^{* * *}$ & $0.848^{* * *}$ \\
\hline QR-VAR $(4,3)$ & 0.056 & 0.154 & 0.235 & 0.483 & 0.732 & 0.948 \\
\hline $\operatorname{VAR}(4)$ & 0.061 & 0.172 & 0.267 & 0.592 & 0.894 & 1.138 \\
\hline relative MSFE & $0.920^{* * *}$ & $0.892^{* * *}$ & $0.879^{* * *}$ & $0.817^{* * *}$ & $0.818^{* * *}$ & $0.832^{* * *}$ \\
\hline \multicolumn{7}{|c|}{ MSFE, short rate (level, $i_{t}$ ) } \\
\hline QR-VAR $(1,1)$ & 0.033 & 0.094 & 0.166 & 0.493 & 0.891 & 1.375 \\
\hline $\operatorname{VAR}(1)$ & 0.036 & 0.112 & 0.207 & 0.615 & 1.086 & 1.616 \\
\hline relative MSFE & $0.907^{* * *}$ & $0.842^{* * *}$ & $0.801^{* * *}$ & $0.801^{* * *}$ & $0.821^{* * *}$ & $0.851^{* * *}$ \\
\hline QR-VAR $(4,3)$ & 0.042 & 0.113 & 0.194 & 0.536 & 1.040 & 1.701 \\
\hline $\operatorname{VAR}(4)$ & 0.051 & 0.143 & 0.236 & 0.628 & 1.154 & 1.770 \\
\hline relative MSFE & $0.821^{* * *}$ & $0.788^{* * *}$ & $0.824^{* * *}$ & $0.853^{* * *}$ & $0.901^{* * *}$ & $0.960 * * *$ \\
\hline \multicolumn{7}{|c|}{ QPS, business cycle $\left(s_{t}\right)$} \\
\hline Univariate model (see (6)) & 0.187 & 0.185 & 0.187 & 0.186 & 0.185 & 0.186 \\
\hline QR-VAR $(1,1)$ & 0.188 & 0.192 & 0.198 & 0.190 & 0.177 & 0.177 \\
\hline relative QPS & 1.001 & 1.039 & 1.054 & 1.025 & 0.957 & 0.950 \\
\hline QR-VAR $(4,3)$ & 0.188 & 0.189 & 0.192 & 0.182 & 0.171 & 0.171 \\
\hline relative QPS & 1.003 & 1.025 & 1.023 & 0.977 & 0.921 & 0.921 \\
\hline
\end{tabular}

Notes: The entries are the MSFE and QPS statistics of different models. Relative MSFEs (QPS) are obtained as dividing the MSFE (QPS) of the QR-VAR model by the MSFE (QPS) of the VAR

(univariate probit) model. The number of simulation replications in the MC forecasting procedure is $N=10000$. In the table, $*, * *, * * *$ denote the $10 \%, 5 \%$ and $1 \%$ level of significance in the test of Clark and West (2007) for equal predictive accuracy between the QR-VAR and the VAR model. 
Table 4: Regime-specific out-of-sample forecast evaluation and business cycle predictability.

\begin{tabular}{|c|c|c|c|c|c|c|}
\hline \multirow[t]{2}{*}{ Model } & \multicolumn{6}{|c|}{ Forecast horizon (months) } \\
\hline & 1 & 2 & 3 & 6 & 9 & 12 \\
\hline \multicolumn{7}{|c|}{$\begin{array}{c}\text { Panel A: Relative MSFE, conditional on the business cycle regime } \\
\text { Term spread }\left(T S_{t}\right)\end{array}$} \\
\hline \multicolumn{7}{|c|}{ Expansion $\left(s_{t}=0\right)$} \\
\hline $\mathrm{QR}-\operatorname{VAR}(1,1) / \operatorname{VAR}(1)$ & 0.910 & 0.862 & 0.809 & 0.762 & 0.760 & 0.800 \\
\hline QR-VAR $(4,3) / \operatorname{VAR}(4)$ & 0.881 & 0.863 & 0.857 & 0.782 & 0.773 & 0.784 \\
\hline \multicolumn{7}{|l|}{ Recession $\left(s_{t}=1\right)$} \\
\hline $\mathrm{QR-VAR}(1,1) / \operatorname{VAR}(1)$ & 1.038 & 1.049 & 1.084 & 1.252 & 1.234 & 1.243 \\
\hline QR-VAR $(4,3) / \operatorname{VAR}(4)$ & 1.027 & 0.991 & 0.945 & 0.974 & 1.047 & 1.136 \\
\hline \multicolumn{7}{|c|}{ Short rate (level, $i_{t}$ ) } \\
\hline \multicolumn{7}{|l|}{ Expansion $\left(s_{t}=0\right)$} \\
\hline $\mathrm{Q} \overline{\mathrm{R}-\operatorname{VAR}(1,1) / \operatorname{VAR}(1)}$ & 0.882 & 0.787 & 0.730 & 0.767 & 0.801 & 0.840 \\
\hline QR-VAR $(4,3) / \operatorname{VAR}(4)$ & 0.757 & 0.748 & 0.826 & 0.895 & 0.924 & 0.973 \\
\hline \multicolumn{7}{|l|}{ Recession $\left(s_{t}=1\right)$} \\
\hline QR-VAR $(1,1) / \operatorname{VAR}(1)$ & 0.961 & 0.929 & 0.907 & 0.870 & 0.865 & 0.880 \\
\hline QR-VAR $(4,3) / \operatorname{VAR}(4)$ & 0.971 & 0.855 & 0.822 & 0.794 & 0.866 & 0.938 \\
\hline \multicolumn{7}{|c|}{ Panel B: AUC, business cycle forecasts $\left(s_{t}\right)$} \\
\hline Univariate model (see (6)) & 0.814 & 0.817 & 0.811 & 0.800 & 0.803 & 0.812 \\
\hline QR-VAR $(1,1)$ & 0.814 & 0.811 & 0.797 & 0.802 & 0.838 & 0.859 \\
\hline QR-VAR $(4,3)$ & 0.814 & 0.813 & 0.805 & 0.818 & 0.849 & 0.866 \\
\hline
\end{tabular}

Notes: In Panel A, the entries are the relative MSFEs between the QR-VAR and VAR models separately for expansion and recession states where the values under unity signify the superiority of the former model. In Panel B, the (out-of-sample) AUCs (Area Under the ROC curve) measure the predictability of the state of the business cycle. Details on the AUC, see, e.g., Berge and Jordà (2011) and Lahiri and Yang (2013). 
Table 5: Out-of-sample forecasting performance of the augmented models and the random walk.

\begin{tabular}{ccccccc}
\hline \hline Model & 1 & 2 & 3 & 6 & 9 & 12 \\
\hline \multicolumn{7}{c}{ MSFE, term spread $\left(T S_{t}\right)$} \\
QR-VAR(1,1) & 0.059 & 0.151 & 0.239 & 0.515 & 0.761 & 0.982 \\
VAR(2)+ipt & 0.057 & 0.158 & 0.248 & 0.549 & 0.851 & 1.092 \\
QR-VAR(1,1)+ipt & 0.060 & 0.156 & 0.246 & 0.559 & 0.828 & 1.054 \\
VAR(2)+emp & 0.058 & 0.155 & 0.228 & 0.465 & 0.712 & 0.931 \\
QR-VAR(1,1)+emp & $0.063^{* *}$ & 0.166 & 0.266 & $0.648^{* *}$ & $0.975^{*}$ & 1.210 \\
RW & $0.063^{*}$ & $0.163^{* *}$ & $0.258^{* *}$ & $0.620^{*}$ & $1.076^{* *}$ & $1.558^{* *}$ \\
\hline \multicolumn{7}{c}{ MSFE, short rate (level, $\left.i_{t}\right)$} \\
QR-VAR(1,1) & 0.033 & 0.094 & 0.166 \\
VAR(2)+ipt & $0.045^{* * *}$ & $0.127^{* * *}$ & $0.212^{* *}$ & 0.493 & 0.895 & 1.375 \\
QR-VAR(1,1)+ipt & 0.035 & 0.102 & 0.189 & 0.600 & 1.111 & 1.108 \\
VAR(2)+emp & $0.049^{* * *}$ & $0.151^{* * *}$ & $0.265^{* * *}$ & $0.680^{* * *}$ & $1.171^{* *}$ & 1.678 \\
QR-VAR(1,1)+emp & $0.041^{* *}$ & $0.119^{*}$ & $0.229^{*}$ & $0.774^{* *}$ & $1.429^{* *}$ & $2.111^{* *}$ \\
RW & $0.044^{* *}$ & $0.128^{* *}$ & $0.236^{* *}$ & $0.702^{*}$ & $1.356^{*}$ & $2.161^{*}$ \\
\hline & \multicolumn{7}{c}{ QPS, business cycle $\left(s_{t}\right)$} \\
QR-VAR(1,1) & 0.188 & 0.192 & 0.198 & 0.190 & 0.177 & 0.177 \\
QR-VAR(1,1)+ipt & 0.183 & 0.188 & 0.193 & 0.190 & 0.177 & 0.173 \\
QR-VAR(1,1)+emp & 0.183 & 0.186 & 0.191 & 0.191 & 0.176 & 0.168 \\
\hline \hline
\end{tabular}

Notes: The entries are the MSFE and QPS statistics in different models. The results of the

QR-VAR $(1,1)$ model given in the first row for each variable are already reported in Table 3 . In the table, ipt (industrial production) and emp (employment) denote the third variable included in $\mathbf{y}_{t}$ (in addition to the term spread and the first-difference of the short-term interest rate) while RW denotes random walk forecasts. In the table. $*, * *, * * *$ denote the $10 \%, 5 \%$ and $1 \%$ level of significance in the Diebold-Mariano (1995) and West (1996) test of equal predictive accuracy between the QR-VAR $(1,1)$ and the model given in the first column (if denoted significant, the QR-VAR outperforms statistically the latter model).

Table 6: Out-of-sample forecasting performance of the MSVAR and VTVAR models (relative MSFEs).

\begin{tabular}{lcccccc}
\hline \hline & \multicolumn{7}{c}{ Forecast horizon } \\
& 1 & 2 & 3 & 6 & 9 & 12 \\
\hline MSVAR $(1)$ & 0.996 & 0.987 & $0.983^{*}$ & $0.841^{* *}$ & $0.685^{* *}$ & $0.567^{* *}$ \\
VTVAR $(2)$ & 0.975 & $0.806^{* * *}$ & $0.749^{* * *}$ & $0.764^{* *}$ & $0.675^{* *}$ & $0.634^{* *}$ \\
\hline & & & Short rate (level, $\left.i_{t}\right)$ \\
MSVAR $(1)$ & 0.929 & $0.875^{*}$ & $0.833^{*}$ & $0.789^{*}$ & $0.731^{*}$ & $0.701^{*}$ \\
VTVAR $(2)$ & $0.669^{* *}$ & $0.502^{* *}$ & $0.453^{* *}$ & $0.477^{* *}$ & $0.413^{* *}$ & $0.390^{* *}$ \\
\hline
\end{tabular}

Notes: The entries are the relative MSFEs obtained as dividing the MSFE of the QR-VAR $(1,1)$ model by the MSFE of the MSVAR(1) and VTVAR(2) model, respectively. In the table. $*, * *, * * *$ denote the $10 \%, 5 \%$ and $1 \%$ level of significance in the Diebold-Mariano (1995) and West (1996) test of equal predictive accuracy between the QR-VAR $(1,1)$ and the model given in the first column (if denoted significant, the QR-VAR outperforms statistically the latter model). Forecasts in the VTVAR model are obtained following the bootstrap-based forecasting method introduced more detail in Teräsvirta et al. (2010, pp. 347-349). 
Table 7: MSFE and QPS statistics of the QR-VAR $(1,1)$ model where the Data Generating Process (DGP) is the QR-VAR $(1,1)$ given in Tables 1 and 2.

\begin{tabular}{ccccccccccc}
\hline \hline & \multicolumn{9}{c}{ MSFE, $y_{1 t}$} & \multicolumn{9}{c}{ MSFE, $y_{2 t}$} \\
$N$ & 1000 & 10000 & 50000 & 1000 & 10000 & 50000 & 1000 & 10000 & 50000 \\
\hline Forecast horizon & \multicolumn{1}{c}{$T=200$} \\
1 & 0.158 & 0.158 & 0.158 & 0.261 & 0.261 & 0.261 & 0.207 & 0.206 & 0.206 \\
2 & 0.400 & 0.399 & 0.399 & 0.277 & 0.276 & 0.276 & 0.200 & 0.199 & 0.199 \\
3 & 0.671 & 0.668 & 0.668 & 0.265 & 0.263 & 0.263 & 0.218 & 0.217 & 0.217 \\
6 & 1.234 & 1.228 & 1.228 & 0.270 & 0.269 & 0.269 & 0.240 & 0.238 & 0.238 \\
9 & 1.535 & 1.530 & 1.529 & 0.278 & 0.276 & 0.276 & 0.257 & 0.257 & 0.257 \\
12 & 1.770 & 1.765 & 1.764 & 0.293 & 0.292 & 0.292 & 0.267 & 0.265 & 0.265 \\
\hline & & \multicolumn{9}{c}{$T=500$} \\
1 & 0.171 & 0.170 & 0.170 & 0.253 & 0.251 & 0.251 & 0.204 & 0.203 & 0.203 \\
2 & 0.444 & 0.442 & 0.442 & 0.277 & 0.276 & 0.276 & 0.205 & 0.204 & 0.204 \\
3 & 0.739 & 0.735 & 0.735 & 0.263 & 0.262 & 0.262 & 0.210 & 0.209 & 0.208 \\
6 & 1.455 & 1.447 & 1.447 & 0.264 & 0.262 & 0.262 & 0.219 & 0.217 & 0.217 \\
9 & 2.070 & 2.061 & 2.060 & 0.265 & 0.264 & 0.264 & 0.225 & 0.224 & 0.224 \\
12 & 2.723 & 2.710 & 2.709 & 0.283 & 0.282 & 0.282 & 0.236 & 0.235 & 0.235 \\
\hline \hline
\end{tabular}

Notes: The entries are based on 5000 realizations. The sample size is 200 or 500 observations $(T=200$ or $T=500)$ and the number of simulation replications in forecast computation is denoted by $N$ where $N=1000,10000$ or 50000 . In simulations from the DGP, following the business cycle periods determined by the NBER, an additional censoring rule is imposed guaranteeing that the sequences of zeros and ones of the values of $s_{t}$ are at least six-period long.

Table 8: The relative MSFE and QPS statistics of the QR-VAR $(1,1)$ relative to the $\operatorname{VAR}(1)$ model and the univariate autoregressive probit model (6).

\begin{tabular}{cccccccc}
\hline \hline & \multicolumn{7}{c}{ Forecast horizon } \\
$T$ & & 1 & 2 & 3 & 6 & 9 & 12 \\
\hline$T=200$ & MSFE, $y_{1 t}$ & 0.971 & 0.930 & 0.884 & 0.773 & 0.704 & 0.641 \\
& MSFE, $y_{2 t}$ & 0.986 & 0.987 & 0.983 & 0.994 & 1.020 & 1.058 \\
& QPS, $s_{t}$ & 1.000 & 0.939 & 0.940 & 0.906 & 0.941 & 0.915 \\
\hline$T=500$ & MSFE, $y_{1 t}$ & 0.964 & 0.947 & 0.931 & 0.899 & 0.850 & 0.832 \\
& MSFE, $y_{2 t}$ & 0.997 & 0.985 & 0.978 & 1.006 & 0.997 & 0.998 \\
& QPS, $s_{t}$ & 1.000 & 1.007 & 1.000 & 0.961 & 0.938 & 0.950 \\
\hline \hline
\end{tabular}

Notes: The number of simulated realizations is 5000 and the number of replications in the forecast computation of the QR-VAR model is $N=10000$. See also the notes to Table 7. 

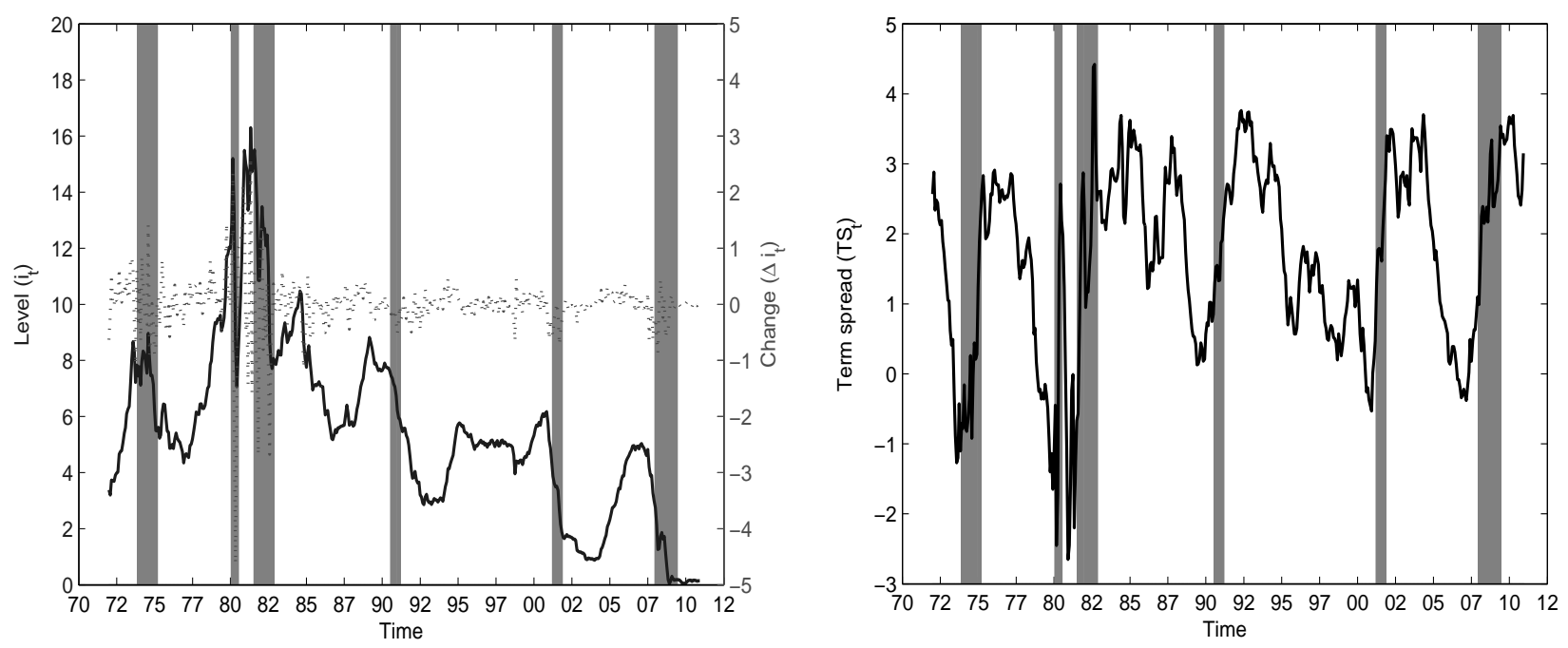

Figure 1: In the left panel, the U.S. short-term interest rate $\left(i_{t}\right)$ and its first difference $\left(\Delta i_{t}\right.$, dashed line) are depicted with the U.S. recession $\left(s_{t}=1\right.$, shaded areas) and expansion periods. The right panel shows the term spread $\left(T S_{t}\right)$.

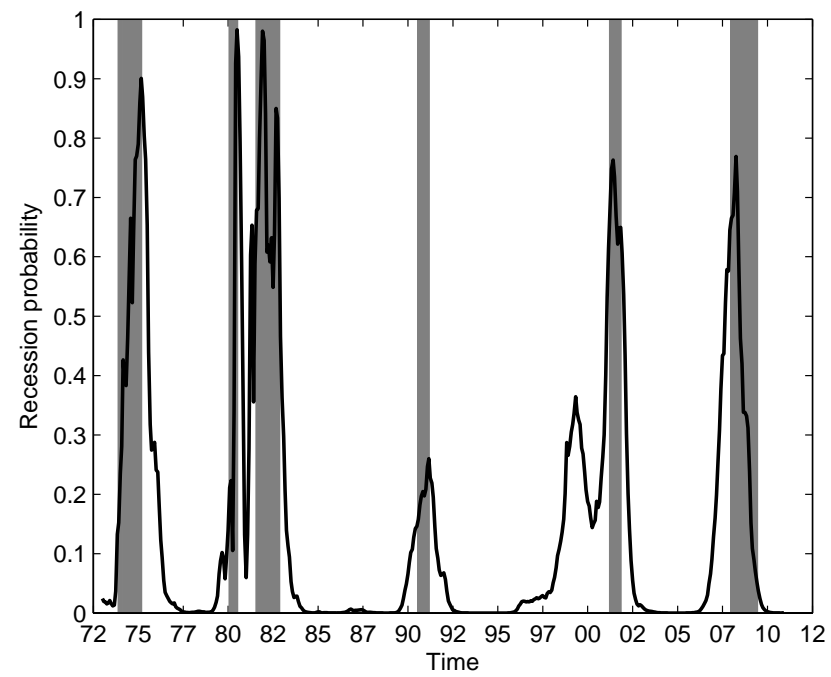

Figure 2: Estimated (in-sample) probability of recession $\left(s_{t}=1\right)$ of the model presented in Table 1. 